\title{
Fundamental limits of electron and nuclear spin qubit lifetimes in an isolated self-assembled quantum dot
}

\author{
George Gillard (iD) ${ }^{1}$, lan M. Griffiths ${ }^{1}$, Gautham Ragunathan ${ }^{1}$, Ata Ulhaq ${ }^{1,3}$, Callum McEwan ${ }^{1}$, Edmund Clarke $\mathbb{D}^{2}$ and \\ Evgeny A. Chekhovich (iD ${ }^{1 凶}$
}

Combining external control with long spin lifetime and coherence is a key challenge for solid state spin qubits. Tunnel coupling with electron Fermi reservoir provides robust charge state control in semiconductor quantum dots, but results in undesired relaxation of electron and nuclear spins through mechanisms that lack complete understanding. Here, we unravel the contributions of tunnelling-assisted and phonon-assisted spin relaxation mechanisms by systematically adjusting the tunnelling coupling in a wide range, including the limit of an isolated quantum dot. These experiments reveal fundamental limits and trade-offs of quantum dot spin dynamics: while reduced tunnelling can be used to achieve electron spin qubit lifetimes exceeding $1 \mathrm{~s}$, the optical spin initialisation fidelity is reduced below $80 \%$, limited by Auger recombination. Comprehensive understanding of electron-nuclear spin relaxation attained here provides a roadmap for design of the optimal operating conditions in quantum dot spin qubits.

npj Quantum Information (2021)7:43; https://doi.org/10.1038/s41534-021-00378-2

\section{INTRODUCTION}

Semiconductor quantum dots (QDs) offer excellent quantum optical properties and well-defined quantum states of individual spins-an attractive combination for quantum information processing devices ${ }^{1}$. Recent proof-of-concept demonstrations with QDs include heralded entanglement of two remote spins ${ }^{2}$, generation of photonic cluster states ${ }^{3}$, spin-controlled photon switching $^{4}$ as well as implementation of electron-nuclear quantum spin interfaces ${ }^{5}$ and nuclear spin quantum computing ${ }^{6}$. The stability of the spin states, measured by their lifetimes, is crucial in all these applications.

Quantum dot is described by a central spin of a single charge (electron or hole) coupled to $N \approx 10^{3}-10^{5}$ nuclear spins via hyperfine interaction ${ }^{7}$. The lack of translational motion combined with the mismatch in electron and nuclear spin energies suppresses relaxation ${ }^{8}$, providing long spin lifetimes required for spin qubits. However, thorough understanding of spin relaxation is complicated by the multitude and complexity of the residual environment couplings, which include electron-phonon interactions $^{9-12}$, quadrupolar coupling of nuclear spins to strain ${ }^{13}$, nuclear spin diffusion, and electron cotunneling ${ }^{14,15}$ arising from proximity of the Fermi reservoir. Moreover, impurity charge traps ${ }^{16,17}$ adjacent to QDs degrade spin qubit lifetimes. Thus, it remains an open question to establish the maximum (intrinsic) spin lifetimes that can be achieved at any given magnetic field and temperature, as opposed to spin relaxation arising from QD device design and imperfections.

Phonon-assisted electron spin relaxation enabled by spin-orbit interaction is a dominant mechanism ${ }^{9,10,18}$ at high magnetic field $B_{z} \gtrsim 2 \mathrm{~T}$, but the limit to electron spin lifetime $T_{1, \mathrm{e}}$ at low fields remains unexplored. In case of nuclear spins, cotunnelingmediated relaxation was identified as dominant mechanism ${ }^{15}$, while direct verification is lacking, since bias control of cotunneling is restricted to a narrow range compatible with single-electron QD state.
Here we study a series of structures where electron cotunneling is controlled directly by the thickness $t_{B}$ of the tunnel barrier separating the dot from the Fermi reservoir. We find that at $B_{z} \gtrsim$ $2 \mathrm{~T}$ and temperatures $\theta>4.2 \mathrm{~K}$ nuclear spin relaxation is dominated by a higher-order process assisted by phonons ${ }^{19,20}$ and noncollinear hyperfine interaction ${ }^{13}$, rather than by cotunneling, which is dominant only at low fields $B_{z} \lesssim 2 \mathrm{~T}$. Electron spin lifetimes exceeding $T_{1, \mathrm{e}}>1 \mathrm{~s}$ are found at $B_{z} \approx 0.4 \mathrm{~T}$, with a fundamental maximum $T_{1, \mathrm{e}} \approx 20 \mathrm{~s}$ estimated for an isolated dot at $\theta=4.2 \mathrm{~K}$, bounded by phonon relaxation and direct hyperfine interaction at high and low magnetic fields, respectively. While coupling to Fermi reservoir degrades $T_{1, \mathrm{e}}$ it is shown to play a crucial role in counteracting Auger recombination ${ }^{21}$ and enabling electron spin initialisation with near-unity fidelity ${ }^{22}$.

\section{RESULTS}

\section{Quantum dot structures and experimental techniques}

Figure 1a sketches conduction band energy profile which is controlled with external bias $V_{\mathrm{S}}$ to tune an InAs QD into Coulomb blockade regime, where it is charged deterministically ${ }^{23}$ with a single electron (1e). The trion state with two electrons and one hole can be accessed through resonant optical excitation, and observed in resonance fluorescence (ResFl). Magnetic field $B_{z}$ along the sample growth axis splits the electron spin-up $|\uparrow\rangle$ and spin-down $|\downarrow\rangle$ energies (Fig. 1b), enabling selective excitation of the optically allowed transition between $|\uparrow\rangle$ electron and the trion $|\uparrow \downarrow \uparrow\rangle$ with a spin-up hole $|\uparrow\rangle$. Weak recombination enabled by hyperfine and heavy-light hole mixing $\beta \ll 1$ can 'shelve' the dot $^{14}$ into $|\downarrow\rangle$ state, quenching ResFl intensity $I_{\text {ResFl }}$ until electron returns to $|\uparrow\rangle$ through a spin flip with rate $\xi_{\uparrow \downarrow}$. Such shelving provides an efficient way both for initialisation and readout of the electron $\operatorname{spin}^{14,22}$. Furthermore, hyperfine interaction $\hat{\mathcal{H}}_{\mathrm{hf}} \propto(\hat{\mathbf{I}} \hat{\mathbf{s}})$ of electron spin $\mathbf{s}$ with nuclear spins I enables electron-nuclear flip-flops, so that repeated electron spin initialisation creates a net

\footnotetext{
${ }^{1}$ Department of Physics and Astronomy, University of Sheffield, Sheffield S3 7RH, UK. ${ }^{2}$ Department of Electronic and Electrical Engineering, University of Sheffield, Sheffield S1 3JD, UK. ${ }^{3}$ Present address: Physics department, SBA School of Science and Engineering, Lahore University of Management Sciences, Sector U, DHA 54792 Lahore, Pakistan.

email: e.chekhovich@sheffield.ac.uk
} 
a

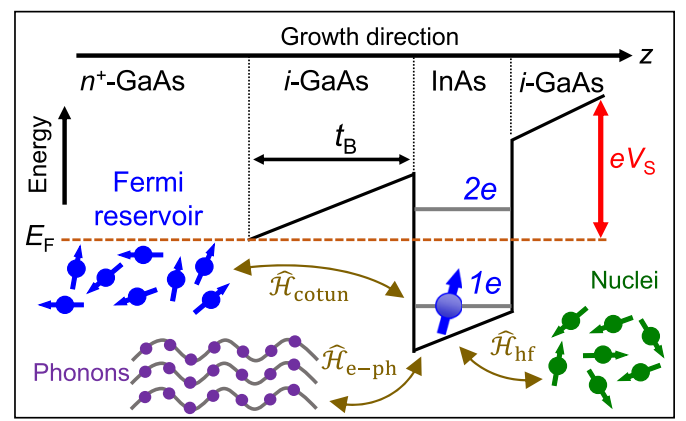

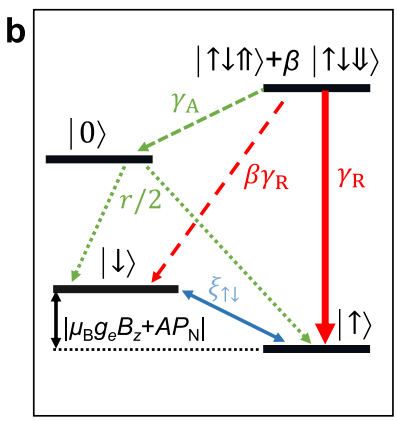

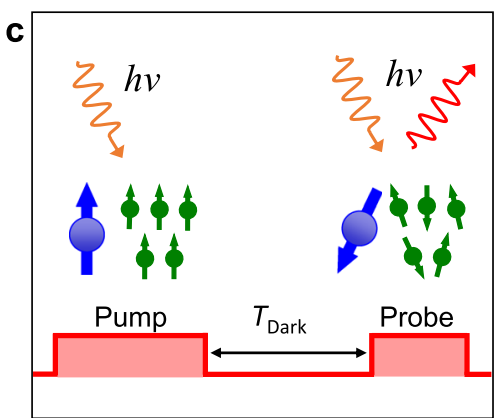

d

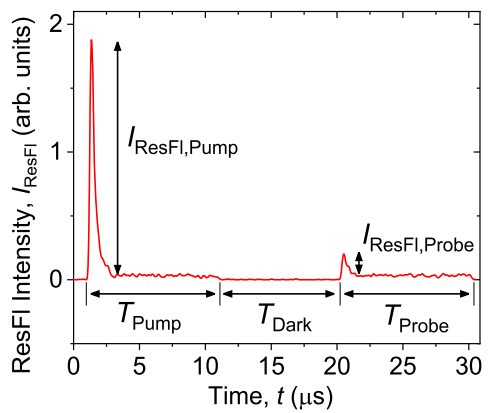

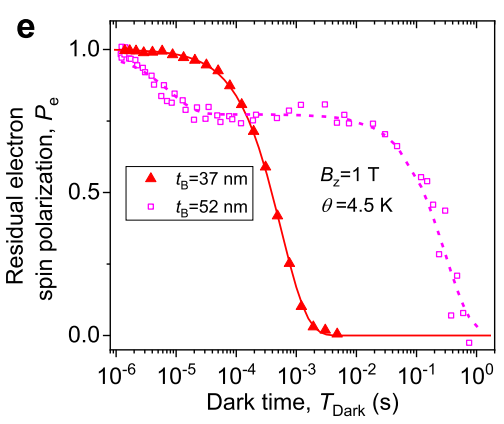

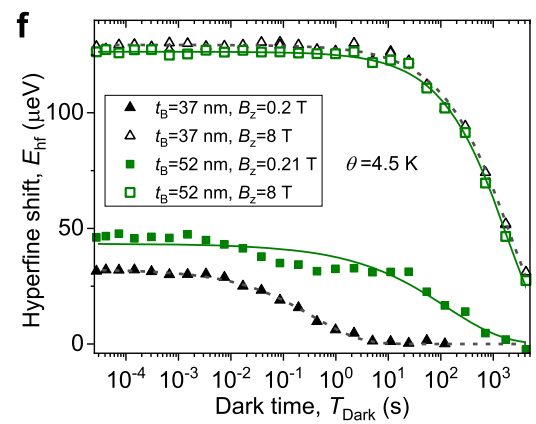

Fig. 1 Electron and nuclear spins in a quantum dot. a Schematic of an InAs quantum dot embedded in a $n-i-S c h o t t k y$ diode structure. Electron spin is coupled to nuclei via hyperfine interaction $\left(\hat{\mathcal{H}}_{\mathrm{hf}}\right)$ and to phonons $\left(\hat{\mathcal{H}}_{\mathrm{e}-\mathrm{ph}}\right)$. Tunnel barrier thickness $t_{\mathrm{B}}$ controls the cotunneling interaction $\left(\hat{\mathcal{H}}_{\text {cotun }}\right)$. Quantum dot charge state is controlled with bias $V_{\mathrm{S}}$, which tunes the energies of one-electron (1e) and two-electron (2e) states with respect to Fermi energy $E_{\mathrm{F}} . \mathbf{b}$ Energy levels of an empty dot $|0\rangle$, electron with spin up $\left(|\uparrow\rangle, s_{z}=+1 / 2\right)$ or down $\left(|\downarrow\rangle, s_{z}=-1 / 2\right)$, and a trion $|\uparrow \downarrow \uparrow\rangle$ with hole moment $j_{z}=+3 / 2$, which has a small admixture $\beta \ll 1$ of a trion $|\uparrow \downarrow \downarrow\rangle$ with opposite hole moment $j_{z}=-3 / 2$. Arrow labels show the rates of radiative recombination $\gamma_{\mathrm{R}}$, Auger recombination $\gamma_{\mathrm{A}}$, recharging $r$, and electron spin flip $\xi_{\uparrow \downarrow}$. Electron spin splitting is due to Zeeman effect $\left(\mu_{\mathrm{B}} g_{\mathrm{e}} B_{\mathrm{z}}\right.$, where $g_{\mathrm{e}}$ is electron $g$-factor and $\mu_{\mathrm{B}}$ is Bohr magneton) and nuclear hyperfine shift $E_{\mathrm{hf}} \propto A_{\mathrm{hf}} P_{\mathrm{N}}$. c Timing diagram of a pump-delay-probe experiment where optical excitation is used to initialise and probe the spins of either the electron or nuclei. d Timeresolved resonance fluorescence (ResFl) in a pump-probe experiment. Resonance fluorescence pulses of intensities $I_{\text {ResFl,Pump }}$ and $I_{\text {ResFl,Probe }}$ indicate electron spin pumping and are used to calculate the residual (i.e. relative to initial) electron spin polarisation $P_{\mathrm{e}}=\left(I_{\mathrm{ResF}, \text { Pump }}-I_{\text {ResFI, }}\right.$ Probe $) / I_{\text {ResFl,Pump }}$ after time $T_{\text {Dark. }}$ e Electron spin decay measured in Coulomb blockade regime as $P_{\mathrm{e}}\left(T_{\text {Dark }}\right)$ in diode sample structures with different $t_{\mathrm{B}}$ (symbols). Lines show exponential (solid) or rate-equation (dashed) fitting. $\mathbf{f}$ Nuclear spin decay obtained by measuring hyperfine shift $E_{\mathrm{hf}}$ as a function of dark time $T_{\text {Dark }}$ in a pump-delay-probe experiment. Lines show fitting with stretched exponential function.

nuclear spin polarisation $P_{\mathrm{N}}$, which can be monitored through optically measured hyperfine shifts $E_{\mathrm{hf}}$ in the splitting of the $|\uparrow\rangle$ and $|\downarrow\rangle$ states 7,17 .

Electron and nuclear spin dynamics are measured using a pump-delay-probe protocol (Fig. 1c). The decay of optically pumped electron [nuclear] spin polarisation over dark period $T_{\text {Dark }}$ is probed by measuring $I_{\text {ResFI }}\left[E_{\mathrm{hf}}\right]$. Figure $1 \mathrm{~d}$ shows an example of time-resolved ResFl, which is used to derive the residual electron spin polarisation $P_{\mathrm{e}}$ after a delay $T_{\text {Dark }}$ (see the "Methods" section). Measurements of $P_{\mathrm{e}}$ at different $T_{\text {Dark }}$ reveal electron spin relaxation (symbols in Fig. 1e), while examples of nuclear spin relaxation $E_{\mathrm{hf}}\left(T_{\text {Dark }}\right)$ are shown in Fig. 1f. Fitting (lines in Fig. $1 e, f)$ is used to derive the intrinsic spin-relaxation rates of electron $\Gamma_{\mathrm{e}}=1 / T_{1, \mathrm{e}}=2 \xi_{\uparrow \downarrow}$ and nuclei $\Gamma_{\mathrm{N}}=1 / T_{1, \mathrm{~N}}$.

\section{Effect of Auger recombination on spin initialisation}

We make a systematic comparison of spin dynamics in a thinbarrier sample $\left(t_{B}=37 \mathrm{~nm}\right.$, Fig. $\left.2 \mathrm{a}-\mathrm{c}\right)$, similar to structures used previously ${ }^{10,14,15}$, and a thick-barrier structure $\left(t_{B}=52 \mathrm{~nm}\right.$, Fig. $2 \mathrm{~d}-\mathrm{f}$ ), approximating a $\mathrm{QD}$ isolated from the Fermi reservoir. Examining the bias dependence of continuous excitation resonance flourescence intensity $I_{\text {ResFl }}$ in a thin-barrier sample, we observe a $1 e$ plateau at $B_{z}=0 \mathrm{~T}$ (triangles in Fig. 2a), while at high $B_{z}$ (circles and squares in Fig. 2a) ResFl is strongly suppressed, indicating spin 'shelving' ${ }^{10,14}$. A striking difference is observed in a thick-barrier sample (Fig. 2d), where fluorescence intensity and spin 'shelving' contrast are reduced (Fig. 2a), which may at first suggest the lack of electron spin initialisation. However, this is ruled out by time-resolved ResFI (e.g. Fig. 1d), which reveals spinpumping fluorescence pulses of similar intensity for all $t_{\mathrm{B}}$. We ascribe the reduction in $I_{\text {ResFl }}$ and the peculiar two-stage electron spin decay in a thick-barrier sample (squares in Fig. 1e) to the Auger process ${ }^{21,24}$, where electron-hole recombination ejects the second electron with a rate $\gamma_{\mathrm{A}}$ (Fig. $1 \mathrm{~b}$ ). Following the ejection, an empty QD does not contribute to ResFl, hence $P_{\mathrm{e}} \approx 1$ observed initially. During $T_{\text {Dark }}$ an unpolarised electron can return from the Fermi reservoir with recharging rate $r$, giving rise to the fast component of the $P_{\mathrm{e}}\left(T_{\text {Dark }}\right)$ decay (squares in Fig. 1e at $T_{\text {Dark }}<$ $100 \mu \mathrm{s})$, whereas the slow component corresponds to resident electron spin relaxation with rate $\Gamma_{\mathrm{e}}$.

Using rate equation modelling (see details in the "Methods" section) of the four-level system shown in Fig. 1b, we find good description of the experiments (dashed line Fig. 1e) and derive $r \approx$ $1.26 \times 10^{5} \mathrm{~s}^{-1}, \Gamma_{\mathrm{e}}=T_{1, \mathrm{e}}^{-1} \approx 3.3 \mathrm{~s}^{-1}$. Importantly, the level $P_{\mathrm{e}} \approx 0.77$ reached after dot recharging $\left(T_{\text {Dark }} \approx 100 \mu \mathrm{s}\right)$ gives a direct measure of the electron spin initialisation fidelity in a thickbarrier sample, revealing the fundamental limitations arising in an isolated $\left(t_{\mathrm{B}} \rightarrow \infty\right) \mathrm{QD}$. In a thin-barrier sample, Auger recombination is counteracted by fast recharging: the resulting spin initialisation fidelity is higher, but can never reach unity. The maximum spin initialisation fidelity is an algebraic function of QD properties such as relaxation rates and heavy-light hole mixing. (The exact expression can be found in Supplementary Note 2.) Analysis shows that fidelity is improved for faster recharging $r$, larger trion mixing $\beta$ and slower spin flip $\xi_{\uparrow \downarrow}$. Conversely, in the limit of infinitely slow recharging $r \rightarrow 0$ spin initialisation becomes 

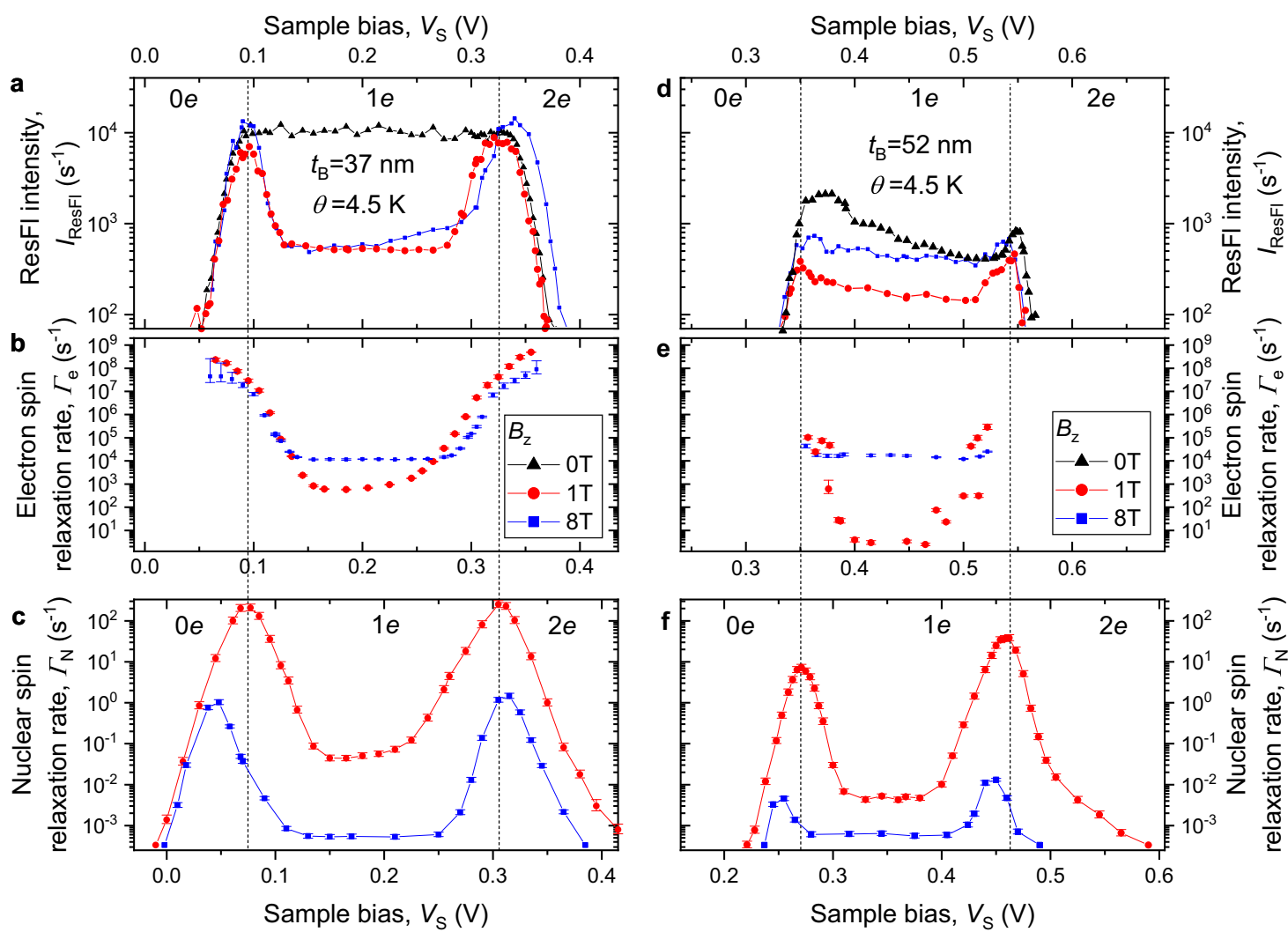

Fig. 2 Bias dependence of electron and nuclear spin dynamics. a Bias dependence of resonance fluorescence (ResFI) intensity $I_{\text {ResFI }}$ in a QD sample with $t_{\mathrm{B}}=37 \mathrm{~nm}$. At $B_{z}=0 \mathrm{~T}$ (triangles) a clear Coulomb blockade (1e plateau) is observed, with $I_{\text {ResFI }}$ decreasing when the dot is emptied $\left(0 e, V_{\mathrm{S}} \lesssim 0.1 \mathrm{~V}\right)$ or filled with a second electron $\left(2 e, V_{\mathrm{S}} \gtrsim 0.33 \mathrm{~V}\right)$. At non-zero $B_{z}=1 \mathrm{~T}$ (circles) and $B_{z}=8 \mathrm{~T}$ (squares) the centre of the $1 e$ plateau is suppressed due to spin 'shelving', leaving two peaks arising from resonant tunnel coupling to the Fermi reservoir. b Bias dependence of the electron spin relaxation rate measured at $B_{z}=1 \mathrm{~T}$ (circles) and $B_{z}=8 \mathrm{~T}$ (squares). $\mathrm{c}$ Bias dependence of the nuclear spin relaxation rate measured for the same QD as in $\mathbf{a}$ and $\mathbf{b}$. Note the offset in the horizontal axis, which is likely due to additional electric fields arising from the optically generated trapped charges near the QD. Vertical dashed lines separate different charge states $0 e, 1 e, 2 e$. $\mathbf{d}-\mathbf{f}$ Same as a-c but for a QD from a thick-barrier sample $\left(t_{\mathrm{B}}=52 \mathrm{~nm}\right)$. All error bars are $95 \%$ confidence intervals.

impossible $\left(P_{\mathrm{e}} \rightarrow 0\right)$, imposing a practical lower limit on the tunnel coupling with the Fermi reservoir.

\section{Fundamental limits of electron spin lifetimes}

Figure 2 shows that electron (Fig. 2b, e) and nuclear (Fig. 2c, f) spin relaxation rates are reduced at the centre of the $1 e$ plateau ${ }^{15,25}$. This Coulomb blockade regime is of most interest, as it corresponds to a stable electron spin qubit, and is examined in more detail in Fig. 3. The dependence of $\Gamma_{\mathrm{e}}$ on magnetic field is well described (solid lines in Fig. 3a) by

$\Gamma_{\mathrm{e}}=\Gamma_{\mathrm{e}, \mathrm{cotun}}+\Gamma_{\mathrm{e}, \mathrm{ph}} B_{\mathrm{z}}^{k_{\mathrm{ph}}}$

where for the field-dependent mechanism assisted by spin-orbit interaction and phonons we find $\Gamma_{\mathrm{e}, \mathrm{ph}} \approx 2.27 \pm 0.48 \mathrm{~s}^{-1} \times \mathrm{T}^{-k_{\mathrm{ph}}}$ and $k_{\mathrm{ph}} \approx 4.1 \pm 0.13$ in both samples. The exponent is in good agreement with $k_{\mathrm{ph}}=4$ predicted $^{26}$ and observed experimentally ${ }^{9}$ for this mechanism in high-temperature regime $\mu_{\mathrm{B}} g_{\mathrm{e}} B_{z} \lesssim k_{\mathrm{B}} \theta$, where phonon thermal occupation factor gives rise to an additional $\propto B_{z}^{-1}$ factor in $\Gamma_{\mathrm{e}}$. This condition is well satisfied for our experiments at $B_{z} \leq 8 \mathrm{~T}, \theta \geq 4.5 \mathrm{~K}$ and typical $g$-factor values $\left|g_{\mathrm{e}}\right| \approx 0.4$. By contrast, in previous studies at $B_{z} \leq 12 \mathrm{~T}, \theta \approx 1 \mathrm{~K}$ the onset of low-temperature regime was observed ${ }^{9}$, where phonon thermal occupation factor is $\approx 1$, resulting in $k_{\mathrm{ph}}=5$.

Cotunneling involves virtual injection of a second electron into the dot, followed by return of a spin-flipped electron to the Fermi reservoir ${ }^{14,27}$. The fitted cotunneling-induced relaxation rate in a thin-barrier $\left(t_{\mathrm{B}}=37 \mathrm{~nm}\right)$ sample $\Gamma_{\mathrm{e}, \text { cotun }} \approx 532 \pm 65 \mathrm{~s}^{-1}=(0.0019 \pm$ $0.0002 \mathrm{~s})^{-1}$ is larger than $\Gamma_{\mathrm{e}, \text { cotun }} \approx(1.65 \pm 0.21 \mathrm{~s})^{-1}$ found for a thicker $t_{\mathrm{B}}=52 \mathrm{~nm}$. Since $\Gamma_{\mathrm{e}, \text { cotun }}$ is field independent, the increase in $\Gamma_{\mathrm{e}}$ at very low $B_{z} \lesssim 0.2 \mathrm{~T}$ (squares in Fig. $3 \mathrm{a}$ ) is likely due to energy-conserving electron-nuclear flip-flops, which become allowed when electron Zeeman energy is comparable to nuclear quadrupolar energy. By extrapolating the phonon (dotted line) and hyperfine (dash-dotted line) mechanisms we roughly estimate the fundamental minimum of the electron spin relaxation rate in an isolated $\left(t_{\mathrm{B}} \rightarrow \infty\right)$ QD as $\Gamma_{\mathrm{e}, \min } \gtrsim(20 \mathrm{~s})^{-1}$, expected to occur at $B_{z} \approx 0.4 \mathrm{~T}$ for $\theta \approx 4.5 \mathrm{~K}$. Similarly slow electron spin relaxation rates were reported in strain-free III-V QDs ${ }^{28}$, although at lower temperatures $\theta<0.1 \mathrm{~K}$.

Figure $3 \mathrm{~b}$ shows $\Gamma_{\mathrm{e}}$ measured in cotunneling-dominated lowfield regime in samples with different barriers. A considerable variation between individual dots for the thin barrier $t_{\mathrm{B}}=37 \mathrm{~nm}$ can be due to random atomic-scale positioning of the individual Si dopants ${ }^{29}$ and $\mathrm{Si}$ segregation ${ }^{30}$ at the interface between tunnel barrier and Fermi reservoir. By contrast, in a thick-barrier sample $\left(t_{\mathrm{B}}=52 \mathrm{~nm}\right)$ the dot is coupled to a large number of dopants, smoothing out atomic-scale variations and leading to consistent $\Gamma_{\mathrm{e}}$.

In low-field regime the barrier thickness $t_{\mathrm{B}}$ controls both the electron spin relaxation rate $\Gamma_{\mathrm{e}}$ and recharging rate $r$, but we find that $r$ exceeds $\Gamma_{\mathrm{e}}$ by approximately five orders of magnitude, as exemplified in Fig. 1e. The recharging of an empty QD is a firstorder tunnelling process, whereas cotunneling in a charged QD is a second-order process ${ }^{31,32}$, which qualititatively explains the difference in rates. Moreover, the charge state of the dot itself may affect the conduction band energy profile, altering the tunnel 

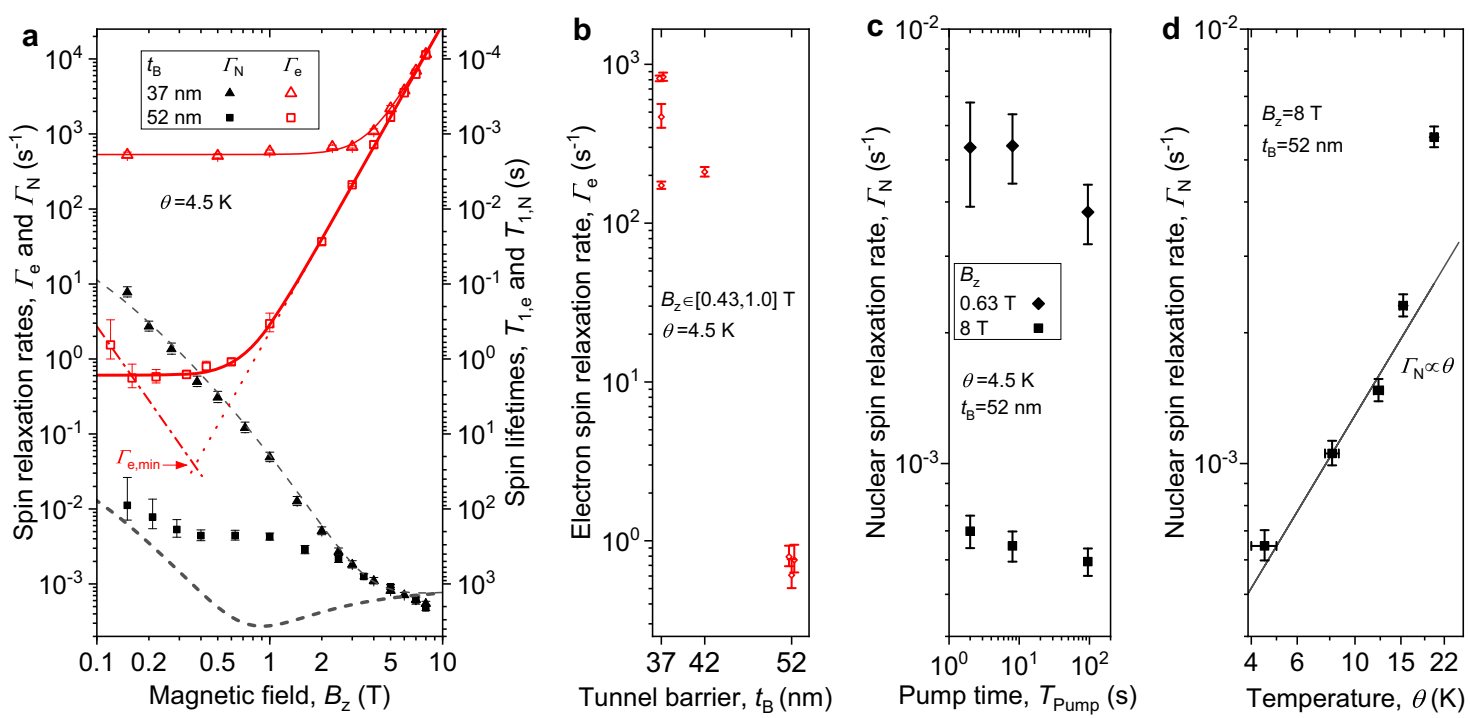

Fig. 3 Electron and nuclear spin dynamics in Coulomb blockade regime. a Magnetic field dependence of the electron $\left(\Gamma_{\mathrm{e}}\right.$, open symbols) and nuclear $\left(\Gamma_{\mathrm{N}}\right.$, solid symbols) spin relaxation rates measured in a thin-barrier (triangles, $\left.t_{\mathrm{B}}=37 \mathrm{~nm}\right)$ and thick-barrier $\left(\mathrm{squares}, t_{\mathrm{B}}=52 \mathrm{~nm}\right.$ ) samples at base temperature $\theta \approx 4.5 \mathrm{~K}$. Tabulated experimental data can be found in Supplementary Note 3. Spin pumping time in nuclear spin relaxation experiments is $T_{\text {Pump }}=8 \mathrm{~s}$. Modelling is shown by the solid (electron) and dashed (nuclei) thin $\left(t_{\mathrm{B}}=37 \mathrm{~nm}\right)$ and thick $\left(t_{\mathrm{B}}=\right.$ $52 \mathrm{~nm}$ ) lines. Dotted and dash-dotted lines show power-law extrapolations of the low-field and high-field regimes to estimate the minimum electron spin relaxation rate $\Gamma_{\mathrm{e}, \min } \approx(20 \mathrm{~s})^{-1}$. b $\Gamma_{\mathrm{e}}$ for several QDs in samples with $t_{\mathrm{B}}=37,42$ and $52 \mathrm{~nm}$ measured in low-field regime $B_{z} \leq 1 \mathrm{~T}$, where spin relaxation is dominated by cotunneling. Small horizontal offsets are added to prevent points from overlapping. c $\Gamma_{\mathrm{N}}$ measured as a function of the pump time in a thick-barrier sample at $B_{z}=0.63 \mathrm{~T}$ (diamonds) and $B_{z}=8 \mathrm{~T}$ (squares). $\mathbf{d} \Gamma_{\mathrm{N}}$ measured as a function of sample temperature in a thick-barrier sample at $B_{z}=8 \mathrm{~T}$ (symbols). Line shows linear dependence. All error bars are $95 \%$ confidence intervals.

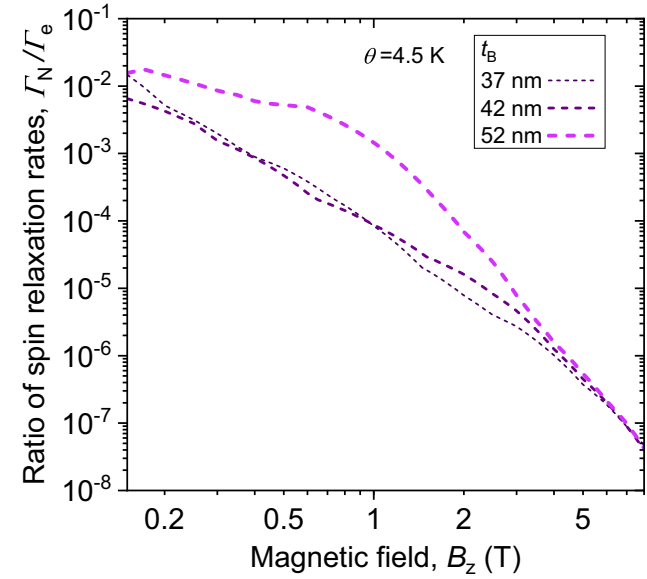

Fig. 4 Ratio of nuclear and electron spin relaxation rates. $\Gamma_{\mathrm{N}} / \Gamma_{\mathrm{e}}$ as a function of magnetic field $B_{z}$ is plotted by taking linear interpolations of the logarithms of experimentally measured nuclear and electron spin relaxation rates. The results are shown for individual quantum dots in three samples with different tunnel barriers $t_{\mathrm{B}}=37,42$ and $52 \mathrm{~nm}$.

coupling. An accurate first-principle quantitative description of the rates would require development of a detailed theoretical model.

\section{Nuclear spin relaxation mechanisms}

The marked difference in nuclear spin relaxation rate $\Gamma_{\mathrm{N}}$ (solid symbols in Fig. 3a) of the two samples at low magnetic fields suggest cotunneling as the dominant channel, whereas at $B_{z} \gtrsim 2 \mathrm{~T}$ cotunneling is negligible. This is in contrast to previous studies under similar conditions $\left(t_{\mathrm{B}}=35 \mathrm{~nm}, B_{z}=5 \mathrm{~T}\right)$, which identified cotunneling and nuclear spin diffusion ${ }^{15}$ as dominant mechanisms. We examine diffusion by varying the spin pumping time (squares in Fig. $3 \mathrm{c}$ ): Taking the difference in $\Gamma_{\mathrm{N}}$ at short and long pumping, the diffusion rate is estimated to be as small as $\Gamma_{\mathrm{N} \text {,diff }} \lesssim$ $10^{-4} \mathrm{~s}^{-1}$ (at $B_{z}=8 \mathrm{~T}$ ). Slow diffusion is due to quadrupolar freezing of nuclear spin flip-flops ${ }^{15,17}$. Moreover, optical pumping through wetting layer states used in this work (as opposed to resonant optical pumping in ref. ${ }^{15}$ ), is likely to polarise nuclear spins not only in the dot but also in its vicinity, further suppressing the diffusion. Thus cotunneling and spin diffusion alone do not account for all the relevant nuclear spin relaxation mechanisms.

To explain the entire $\Gamma_{\mathrm{N}}\left(B_{\mathrm{z}}\right)$ dependence, we treat the average spin of the QD electron as a random process. Uniquely for selfassembled QDs, noncollinear hyperfine interaction permits nuclear spin relaxation without electron spin flip $^{13}$-this mechanism is expected to be more efficient than direct electron-nuclear spin flips ${ }^{15}$. The transition rate $\Gamma_{\mathrm{N}, i j}$ between states $|i\rangle$ and $|j\rangle$ of a single nuclear spin is proportional to spectral power density ${ }^{33}$ of the fluctuating electron spin $s_{z}(t)$ at the nuclear spin transition frequency $v_{i j}$. Using first-order perturbation theory ${ }^{13}$ we have

$$
\Gamma_{\mathrm{N}, i j}=\left(\frac{A_{\mathrm{hf}}}{N \hbar}\right)^{2} \frac{2\left|M_{i j}\right|^{2} \tau_{\mathrm{e}}}{1+4 \pi^{2} \tau_{\mathrm{e}}^{2} v_{i j}^{2}}
$$

where $A_{\mathrm{hf}}$ is the hyperfine constant, $M_{i j}=\left\langle i\left|\hat{I}_{z}\right| j\right\rangle$ is the matrix element of the nuclear spin operator $\hat{l}_{z}$, and electron spin correlation time is approximated by $\tau_{\mathrm{e}} \approx 1 / \Gamma_{\mathrm{e}}$. This model describes a higher-order nuclear spin relaxation process, mediating by electron spin relaxation, which in turn is dominated by phonons at high fields or cotunneling at low fields. In the high field limit $\left|M_{i j}\right|^{2} \propto B_{z}^{-2}, \Gamma_{\mathrm{e}} \propto B_{z}^{4}$ and $v_{i j}^{2} \propto B_{z}^{2}$ (see the "Methods" section) leading to $\Gamma_{\mathrm{N}} \propto$ const, which agrees with the weak field dependence of $\Gamma_{\mathrm{N}}$ observed for both samples at $B_{z} \gtrsim 4 \mathrm{~T}$ (solid symbols in Fig. 3a). Moreover, temperature dependence at high field (Fig. $3 \mathrm{~d}$ ) is close to linear $\Gamma_{\mathrm{N}} \propto \theta$ at $\theta \lesssim 15 \mathrm{~K}$, matching the $\Gamma_{\mathrm{e}} \propto \theta$ dependence ${ }^{34}$ of the underlying phonon-mediated electron spin relaxation process. Superlinear growth of $\Gamma_{\mathrm{N}}$ at $\theta \gtrsim 15 \mathrm{~K}$ is likely due to two-phonon processes ${ }^{26,35}$, with scaling predicted to range between $\propto \theta^{2}$ and $\propto \theta^{9}$.

For quantitative description (see the "Methods" section) we use experimentally measured $\Gamma_{\mathrm{e}}$ and estimate $v_{i j}$ and $M_{i j}$ from 
magnetic resonance spectra ${ }^{36,37}$. The results (dashed lines in Fig. $3 a)$ are in good agreement for the thin barrier $\left(t_{B}=37 \mathrm{~nm}\right)$, where electron correlation time is short. The discrepancy for the thickbarrier sample $\left(t_{\mathrm{B}}=52 \mathrm{~nm}\right)$ is most prominent at $B_{z} \lesssim 2.5 \mathrm{~T}$, revealing the limitations of the electron-spin fluctuation model (Eq. (2)) in the previously unexplored regime of a nearly isolated long-lived electron spin. To examine the cause, we note that $\tau_{\mathrm{e}}^{2} v_{i j}^{2} \gg 1$ except for possible quadrupolar anti-crossings of the nuclear spin levels ${ }^{37,38}$, so that Eq. (2) can be rewritten as

$\Gamma_{\mathrm{N}, i j} \tau_{\mathrm{e}}=\left(\frac{A_{\mathrm{hf}}\left|M_{i j}\right|}{\sqrt{2} \pi N \hbar v_{i j}}\right)^{2}$

The right side of this equation is a function of magnetic field and quantum dot structural properties, such as chemical composition and strain inhomogeneity, but it does not depend on tunnel coupling to Fermi reservoir. If $\tau_{\mathrm{e}}=1 / \Gamma_{\mathrm{e}}$, the equation predicts independence of $\Gamma_{\mathrm{N}} / \Gamma_{\mathrm{e}}$ on tunnel barrier. This is seen to be the case in Fig. 4 for samples with $t_{\mathrm{B}}=37$ and $42 \mathrm{~nm}$, whereas the thick barrier sample $\left(t_{\mathrm{B}}=52 \mathrm{~nm}\right)$ shows excessive $\Gamma_{\mathrm{N}} / \Gamma_{\mathrm{e}}$.

The exact reason for the increased $\Gamma_{\mathrm{N}} / \Gamma_{\mathrm{e}}$ in the thick barrier sample is not clear. One likely possibility is additional nuclear spin relaxation mechanisms where hyperfine interaction fluctuates without electron spin flips, resulting in $\tau_{\mathrm{e}}<1 / \Gamma_{\mathrm{e}}$. For example, modulation of the hyperfine interaction can occur through electron wavefunction density shifts, arising from fluctuating electric fields of the itinerant carriers in the Fermi reservoir ${ }^{33,39}$, or charge traps ${ }^{16}$. Charge noise in the studied structures is indeed present and evidenced, e.g. by fluctuating electron spin relaxation rates at the edges of $1 e$ plateau (circles in Fig. 3e). Future experiments using, e.g. bias modulation spectroscopy may elucidate the roles of different nuclear spin relaxation mechanisms and lead to more accurate theoretical models. Further improvements to nuclear spin relaxation description can be sought through a microscopic model that takes into account quadrupolar anti-crossings of the individual nuclear spin levels ${ }^{37,38}$, which may accelerate relaxation and reenable frozen spin diffusion. A contribution of direct nuclear-phonon interaction ${ }^{33,40}$ is also possible, as its rate $\Gamma_{\mathrm{N}} \approx 10^{-4}-10^{-3} \mathrm{~s}^{-1}$ is comparable to the lowest $\Gamma_{\mathrm{N}}$ observed here in electron-charged QDs.

\section{DISCUSSION}

Experiments presented here establish a comprehensive picture of electron-nuclear spin relaxation in self-assembled QDs in a wide range of practically accessible conditions. Present experiments require $B_{z} \gtrsim 0.15 \mathrm{~T}$ to initialise the spins ${ }^{22}$ and resolve the Zeemansplit optical transitions for spin probing. Extension to lower fields could shed light on the less explored regime where electron spin relaxation abruptly slows down from $\Gamma_{\mathrm{e}} \approx 10^{9} \mathrm{~s}^{-1}$ at zero field ${ }^{8,41}$ to $\Gamma_{\mathrm{e}} \approx 1 \mathrm{~s}^{-1}$ observed here at $0.15 \mathrm{~T}$. For the practically interesting range $B_{z} \gtrsim 0.15 \mathrm{~T}$, electron spin relaxation is fundamentally limited by phonon coupling, which is similar in other types of QDs. Hence, electron spin lifetimes exceeding $1 \mathrm{~s}$ should be achievable in $\mathrm{GaAs}$ electrostatic $^{28}$ and epitaxial ${ }^{42}$ QDs, as well as in II-VI QDs ${ }^{43,44}$. By contrast, nuclear spin relaxation studied here is specific to selfassembled III-V QDs, and is governed by noncollinear hyperfine interaction. All experiments here were conducted in Faraday geometry, whereas noncollinear interaction is expected to be even stronger for magnetic field tilted away from the sample growth axis ${ }^{37}$, which may lead to faster nuclear spin relaxation in Voigt geometry. The techniques employed here, can also be applied to establish the less explored fundamental limits of nuclear spin dynamics in electron-charged strain-free $\mathrm{QDs}^{28,42}$, where noncollinear interaction will be small, but nuclear spin diffusion might be more prominent.

\section{METHODS}

\section{Samples and experimental techniques}

The samples are low-density InAs self-assembled QDs ( $\lesssim 1$ QD per $\mu \mathrm{m}^{2}$ ) grown on a GaAs substrate. The dot layer is positioned at the centre of a $\lambda$ / 2 optical cavity formed by a bottom Bragg mirror consisting of $15 \mathrm{GaAs} /$ AlAs pairs and a top reflector with 2 pairs (estimated quality factor $Q \approx 60$ ). Cavity mode is centred at $950 \mathrm{~nm}$, which matches the long-wavelength tail of the QD wavelength distribution. The Fermi reservoir is formed by a doped GaAs layer (Si concentration of $1.1 \times 10^{18} \mathrm{~cm}^{-3}$, thickness $\approx 80 \mathrm{~nm}$ ). The doped layer is located beneath QDs and is separated by a GaAs layer of thickness $t_{\mathrm{B}}=37-52 \mathrm{~nm}$, depending on the structure. Each sample is processed into a Schottky diode structure with an Au/(In-Ge) ohmic back contact ${ }^{45}$ annealed from the top surface, and a $5 \mathrm{~nm}$-thick semitransparent Ti Schottky top contact. External bias is applied to the top contract and controls the charge states of QDs. In order to form an electron spin qubit, the dot is charged deterministically with one electron (1e). This is achieved by tuning the energy of the $1 e$ state to $\approx 10 \mathrm{meV}$ below the Fermi energy $E_{\mathrm{F}}$ (Fig. 1a), while the two-electron (2e) state remains depopulated, since its energy exceeds $E_{\mathrm{F}}$ by $\approx 10 \mathrm{meV}$, which is $\gtrsim 20$ times the thermal energy $k_{\mathrm{B}} \theta$ at liquid helium temperature $\theta \approx 4.2 \mathrm{~K}$ (Boltzmann constant $k_{\mathrm{B}} \approx 86.17 \mu \mathrm{eV}$ $\left.\mathrm{K}^{-1}\right)$. The dot is then charged by an electron tunnelling from the Fermi reservoir.

The sample is mounted in a bath cryostat equipped with a superconducting coil producing magnetic field up to $8 \mathrm{~T}$ in Faraday geometry (field parallel to sample growth direction and optical axis $z$ ). An aspheric lens mounted near the sample is used for optical excitation of the QD and for light collection. Photoluminescence (PL) spectroscopy (see Supplementary Fig. 1) is used for initial QD characterisation. In nuclear spin dynamics experiments the dot is excited using diode lasers operating at $850 \mathrm{~nm}$ (resonant with InGaAs wetting layer). Nuclear spin polarisation (cooling) is achieved with a circularly polarised high power $(\gtrsim 100$ times the power of ground state exciton saturation) laser, with typical pump pulse duration of $T_{\text {Pump }} \approx 8 \mathrm{~s}$. A short $\left(T_{\text {Probe }} \approx 10 \mathrm{~ms}\right.$ ) low power (approximately corresponding to ground state exciton saturation) probe pulse is used to excite $\mathrm{PL}$, which is then analysed on a double grating spectrometer to derive the hyperfine shifts $E_{\mathrm{hf}}$ in the splitting of a QD Zeeman doublet. The relaxation of the nuclear spin polarisation is derived by measuring $E_{\mathrm{hf}}$ in the probe as a function of delay $T_{\text {Dark }}$ between the pump and the probe. The resulting $E_{\mathrm{hf}}\left(T_{\text {Dark }}\right)$ dependencies (e.g. Fig. 1f) are fitted with stretched or compressed exponentials $\propto \mathrm{e}^{-\left(T_{\text {Dark }} / T_{1, \mathrm{~N}}\right)^{\eta}}$, where $\eta$ is the parameter describing stretching $(\eta<1)$ or compression $(\eta>1)$.

In ResFl experiments the dot is excited with a linearly polarised singlemode tunable diode laser. The scattered laser is rejected using crosspolarised detection ${ }^{46}$, and the collected fluorescence is directed to an avalanche photodiode detector, whose photon-counting pulses are measured with a pulse counter and a digital oscilloscope. Typical linewidths measured in continuous excitation ResFl spectra on a negatively charged trion are $\approx 0.5 \mathrm{GHz}$ at low power (non-saturating excitation). Electron spin initialisation at finite magnetic field is witnessed through ResFI intensity $I_{\text {ResFl, }}$ which is significantly reduced when electron is initialised into the $|\downarrow\rangle$ state, taking the dot out of resonance with optical driving of the $|\uparrow\rangle \leftrightarrow|\uparrow \downarrow \uparrow\rangle$ transition (compare squares and circles with triangles in Fig. 2a). Electron spin relaxation is accelerated and spin shelving is destroyed when the bias is tuned to the level where Fermi reservoir is resonantly tunnel-coupled with $1 e\left(V_{\mathrm{s}} \approx 0.1 \mathrm{~V}\right.$ in Fig. $\left.2 \mathrm{a}\right)$ or $2 e$ $\left(V_{S} \approx 0.33 \mathrm{~V}\right.$ in Fig. 2a) quantum dot state, resulting in two peaks in the $I_{\text {ResFI }}\left(V_{s}\right)$ dependence (squares and circles in Fig. 2a).

In electron spin dynamics measurements pulsing of the resonant laser is achieved with acousto-optical modulators providing on/off ratio better than $10^{7}$. The power of the pump and probe pulses is close to ResFI saturation conditions and typical duration is $T_{\text {Pump }} \approx T_{\text {Probe }} \approx 5-10 \mu$ s, which is significantly longer than the spin pumping time. As a result, timeresolved ResFl exhibits short pulses (Fig. 1d) with amplitudes $I_{\text {ResFl,Pump }}$ and

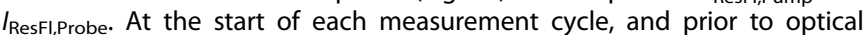
pump pulse, the bias is adjusted for resonant electron tunnelling in order to counteract optical nuclear spin pumping and depolarise the electron (see further details in Supplementary Note 1). The rising edge of the fluorescence pulse corresponds to the rise time of the laser intensity in a pulse that pumps the initially depolarised electron. The exponentially decaying falling edge of the fluorescence pulse traces the gradual shelving (initialisation) of the dot into the $|\downarrow\rangle$ electron spin state. The loss of electron spin polarisation during the delay $T_{\text {Dark }}$ results in partial recovery of the fluorescence intensity measured in the probe pulse. The residual polarisation $P_{\mathrm{e}}$ (i.e. electron spin polarisation at the start of the probe 
normalised by polarisation at the end of the pump pulse) is then derived as $P_{\mathrm{e}}=\left(I_{\text {ResFl,Pump }}-I_{\text {ResFl,Probe }}\right) / I_{\text {ResFl,Pump }}$. This way complete loss of electron polarisation $\left(P_{\mathrm{e}}=0\right)$ is observed as $I_{\text {ResFI,Probe }}=I_{\text {ResFI,Pump, }}$ while $I_{\text {ResFI,Probe }}$ $=0$ implies no loss $\left(P_{\mathrm{e}}=1\right)$ or Auger recombination that empties the dot. By measuring $P_{\mathrm{e}}$ at different $T_{\text {Dark }}$ the decay of electron spin polarisation is obtained as shown in Fig. 1e by the symbols.

Unless stated otherwise, all error estimates in the text and error bars in figures are $95 \%$ confidence intervals.

\section{Modelling of the electron spin relaxation dynamics}

We simulate the dynamics of the four-level system shown in Fig. $1 \mathrm{~b}$ using a simplified noncoherent rate equation model. The relaxation rates of all possible transitions are shown in Fig. $1 \mathrm{~b}$, and when resonant optical pumping is present we add a transition $|\uparrow\rangle \rightarrow|\uparrow \downarrow \uparrow\rangle$ with rate $P$. We assume symmetric rates in electron spin flips $|\uparrow\rangle \leftrightarrow|\downarrow\rangle$, which is justified when electron Zeeman energy is smaller than the thermal energy $k_{B} \theta$. The system of first-order differential rate equations is

$$
\frac{\partial}{\partial t}\left(\begin{array}{c}
p_{|0\rangle}(t) \\
p_{|\uparrow\rangle}(t) \\
p_{|\downarrow\rangle}(t) \\
p_{|\uparrow \downarrow \uparrow\rangle}(t)
\end{array}\right)=\mathbf{R}\left(\begin{array}{c}
p_{|0\rangle}(t) \\
p_{|\uparrow\rangle}(t) \\
p_{|\downarrow\rangle}(t) \\
p_{|\uparrow \downarrow \uparrow\rangle}(t)
\end{array}\right)
$$

$\mathbf{R}=\left(\begin{array}{cccc}-r & 0 & 0 & \gamma_{\mathrm{A}} \\ r / 2 & -P-\xi_{\uparrow \downarrow} & \xi_{\uparrow \downarrow} & \gamma_{\mathrm{R}} \\ r / 2 & \xi_{\uparrow \downarrow} & -\xi_{\uparrow \downarrow} & \beta \gamma_{\mathrm{R}} \\ 0 & P & 0 & -(1+\beta) \gamma_{\mathrm{R}}-\gamma_{\mathrm{A}}\end{array}\right)$

In simulations we set $\gamma_{R}=10^{9} \mathrm{~s}^{-1}$, which is typical for $\ln A s / G a A s ~ Q D s{ }^{47}$. In order to simulate the pump-delay-probe experiment we use initial population probabilities $\left(p_{|0\rangle}, p_{|\uparrow\rangle}, p_{|\downarrow\rangle}, p_{|\uparrow \downarrow \uparrow\rangle}\right)=(0,1 / 2,1 / 2,0)$, and propagate the equations numerically over pump pulse (with $P \neq 0$ ), dark delay $(P=0)$, and probe $(P \neq 0)$. The values of $\gamma_{\mathrm{A}}, P, \beta, r, \xi_{\uparrow \downarrow}$ are used as fitting parameters. The evolution of the trion state population $p_{|\uparrow \downarrow \uparrow\rangle}(t)$ reproduces the experimentally measured time-resolved ResFI (e.g. Fig. $1 \mathrm{~d})$. The simulated $p_{|\uparrow| \uparrow\rangle}(t)$ traces are integrated over pump and probe intervals and are used to calculate the residual electron polarisation $P_{\mathrm{e}}$ in the same way $I_{\text {ResFl,Pump }}$ and $I_{\text {ResFl,Probe }}$ are used to calculate $P_{\mathrm{e}}$ from experimental data (e.g. Fig. 1e). The fitting parameters are adjusted to achieve two-objective optimisation: one objective is to minimise the root mean square difference between simulated and experimental $P_{\mathrm{e}}\left(T_{\text {Dark }}\right)$ traces, the other objective is to match the characteristic exponential time in the falling edge of the ResFl intensity produced by the pump pulse. An example of the Pareto-optimal fitted $P_{\mathrm{e}}\left(T_{\text {Dark }}\right)$ is shown by the dashed line in Fig. 1e, in good agreement with experiment (squares). The calculated falling edge time $(87 \mathrm{~ns})$ is also in good agreement with the experimental value $\approx 91 \mathrm{~ns}$.

From fitting we find $r \approx 1.26 \times 10^{5} \mathrm{~s}^{-1}$ (95\% confidence interval [0.47 $\times$ $\left.\left.10^{5} \mathrm{~s}^{-1}, 3.34 \times 10^{5} \mathrm{~s}^{-1}\right]\right)$ and $\xi_{\uparrow \downarrow} \approx 1.65 \mathrm{~s}^{-1}\left[1.16 \mathrm{~s}^{-1}, 2.34 \mathrm{~s}^{-1}\right]$, which correspond to the characteristic timescales of the fast and slow components, respectively, in the two-stage decay (squares in Fig. 1e). For Auger rate we obtain $\gamma_{\mathrm{A}} \approx 1.09 \times 10^{7} \mathrm{~s}^{-1}\left[0.66 \times 10^{7} \mathrm{~s}^{-1}, 3.11 \times 10^{7} \mathrm{~s}^{-1}\right]$. This is approximately five times higher than $\gamma_{\mathrm{A}} \approx 0.23 \times 10^{7} \mathrm{~s}^{-1}$ reported previously from time-resolved ResFl experiments ${ }^{21}$. The discrepancy could be due to the difference in QD structures, high optical pump power used in our experiments, limitations of a non-coherent rate equation model, and uncertainty in the fitted parameters. The uncertainty is increased by the interdependence of the fitting parameters $\gamma_{\mathrm{A}}, P, \beta, r, \xi_{\uparrow \downarrow}$, which is inevitable since five parameters are used to fit essentially four degrees of freedom (fast and slow rates of the two stage $P_{\mathrm{e}}\left(T_{\text {Dark }}\right)$ decay, $P_{\mathrm{e}}$ following fast decay and the characteristic time of the falling edges in fluorescence pulses). This uncertainty can also be understood to arise from the limited information provided by the ResFl measurement, which does not distinguish between spin shelving into the $|\downarrow\rangle$ state and Auger recombination into the $|0\rangle$ state, restricting the ability to monitor the full dynamics of the four-level system. The remaining best fit values are $P \approx 2.5 \times 10^{9} \mathrm{~s}^{-1}\left[0.34 \times 10^{9} \mathrm{~s}^{-1}, 3.16 \times\right.$ $\left.10^{9} \mathrm{~s}^{-1}\right]$ and $\beta \approx 5.2 \times 10^{-3}\left[0.75 \times 10^{-3}, 20.1 \times 10^{-3}\right]$.

\section{Modelling of the nuclear spin relaxation rate}

We start by noting that at all magnetic fields used in this study $\left(B_{z}=\right.$ $0.15-8 \mathrm{~T}$ ) nuclear spin relaxation in an empty dot $(0 e)$ is at least an order of magnitude slower than at the centre of the $1 e$ charging plateau. (In $0 e$ regime we measure $\Gamma_{\mathrm{N}} \approx 6.0 \times 10^{-4} \mathrm{~s}^{-1}$ at $B_{z}=0.15 \mathrm{~T}$, which reduces at higher fields below the minimum measurable level of $\Gamma_{\mathrm{N}}<10^{-4} \mathrm{~s}^{-1}$.) This suggests that electron is the dominant mediator of nuclear spin relaxation in a Coulomb blockade regime (1e). Electron-nuclear coupling is given by the hyperfine Hamiltonian

$\hat{\mathcal{H}}_{\mathrm{hf}}=\sum_{k} A_{\mathrm{hf}, k}\left(\hat{s}_{x} \hat{I}_{x, k}+\hat{s}_{y} \hat{l}_{y, k}+\hat{s}_{z} \hat{I}_{z, k}\right)$

where the summation goes over all nuclei, $A_{\mathrm{hf}, k}$ is the hyperfine constant of the $k$ th nucleus, $\left(\hat{s}_{x}, \hat{s}_{y}, \hat{s}_{z}\right)$ are the components of the electron spin-1/2 operator and $\left(\hat{l}_{x, k}, \hat{l}_{y, k}, \hat{l}_{z, k}\right)$ are the components of the spin operator of the $k$ th nucleus. The flip-flop term $\propto\left(\hat{s}_{x} \hat{l}_{x}+\hat{s}_{y} \hat{l}_{y}\right)$ of this interaction permits spin exchange between nuclear spin I and electron spin $\mathbf{s}$, but at sufficiently large magnetic field, where electron Zeeman energy significantly exceeds nuclear Zeeman and quadrupolar spin splitting, such flip-flops are strongly suppressed. For a depolarised nuclear spin ensemble in an InAs QD, this threshold field would be on the order of $0.03 \mathrm{~T}$, corresponding to statistical fluctuation $\propto A_{\mathrm{hf}, k} \sqrt{N}$ of the nuclear hyperfine field acting on the electron. However, in self-assembled QDs the principal strain axis is generally misaligned ${ }^{36,37}$ from the growth axis $z$, resulting in nuclear eigenstates which are superpositions of $\hat{l}_{z}$ eigenstates. Under these conditions nuclear spin states are mixed by the non-flip-flop part of the hyperfine interaction $A_{h f, k} \hat{l}_{z, k} \hat{s}_{z}$. This noncollinear interaction enables transitions between nuclear spin states $|i\rangle$ and $|j\rangle$ without transfer of spin to the electron. Using first-order perturbation and Weisskopf-Wigner approximation one arrives to Eq. (2) for nuclear spin relaxation rate between the pair of states $|i\rangle$ and $|j\rangle$, where we have assumed the same hyperfine constant $A_{\mathrm{hf}, \mathrm{k}}=A_{\mathrm{hf}} / \mathrm{N}$ for all nuclei.

In order to calculate matrix element $M_{i j}$ of $\hat{I}_{z}$ we consider spin $I=3 / 2$ and assume that the principal component of electric field gradient is characterised by quadrupolar shift frequency $v_{\mathrm{Q}}$, and is tilted by angle $a$ from the $z$-axis. Using first-order perturbation approach we calculate $M_{i j}$ in the opposite limits of small magnetic field $\left(\gamma_{N} B_{z} \ll v_{Q}\right.$, where $\gamma_{N}$ is the nuclear gyromagnetic ratio) and high magnetic field $\left(\gamma_{\mathrm{N}} B_{z} \gg v_{\mathrm{Q}}\right)$. For each individual nucleus the pair $(|i\rangle,|j\rangle)$ with the largest off-diagonal matrix element is then selected leading to:

$$
\begin{aligned}
& |M|_{B_{z} \rightarrow 0}^{2}=\frac{3}{4} \cos ^{2} a \sin ^{2} a \\
& |M|_{B_{z} \rightarrow \infty}^{2}=\frac{3 \pi v_{Q}^{2}}{8 \gamma_{N}^{2} B_{z}^{2}} \sin ^{4} a,
\end{aligned}
$$

For intermediate fields we interpolate the matrix element with a monotonic function: $\left|M\left(B_{z}\right)\right|^{2}=1 /\left(|M|_{B_{z} \rightarrow 0}^{-2}+|M|_{B_{z} \rightarrow \infty}^{-2}\right)$, and the nuclear spin transition frequency is taken to be $v_{i j}^{2}=\left(\gamma_{\mathrm{N}} / 2 \pi\right)^{2}\left(B_{z}^{2}+B_{z, \min }^{2}\right)$, where non-zero $B_{z, \min }$ reflects the fact that at $B_{z}=0$ the spin states are split by nuclear quadrupolar effects. This model is a simplification since nuclear spin levels (anti)cross ${ }^{38}$ at $B_{z} \approx 2 \pi v_{\mathrm{Q}} / \gamma_{N}$, where nucleus experiences a nearly zero effective magnetic field. The perturbative approach breaks down as $M_{i j}$ is enhanced and $v_{i j} \approx 0$ at these anticrossing points (typically occurring at $\left.B_{z} \approx 1 \mathrm{~T}\right)^{37}$, hence the introduction of $B_{z, \min }$ which softens the singularities in Eq. (2). While spin relaxation would be enhanced in such resonant nuclei, it would also prevent their optical polarisation, thus we effectively neglect their contribution to the overall measured nuclear spin decay in our simplified model.

In order to calculate the nuclear spin decay rate according to Eq. (2), we take $\tau_{\mathrm{e}}=\Gamma_{\mathrm{e}}^{-1}$, equivalent to assuming that electron spin flips are the only source of noise acting on the nuclear spins. For $\Gamma_{\mathrm{e}}$ we use Eq. (1) taking best fit parameters for each QD sample. Based on NMR spectroscopy of similar QDs ${ }^{36,37}$, we model strain inhomogeneity within the quantum dot by considering a uniformly distributed quadrupolar shift $v_{\mathrm{Q}} \in[0,16] \mathrm{MHz}$ with principal axis uniformly distributed on a part of a sphere with $a \in\left[0^{\circ}\right.$, $76^{\circ}$. The large values of $a \approx 76^{\circ}$ account for As nuclei, whose quadrupolar shifts are dominated by atomic scale disorder, arising from $\mathrm{Ga}$ and In alloying $^{36}$. The gyromagnetic ratio is also varied uniformly $\gamma_{N} / 2 \pi \in[7.4,9.2]$ $\mathrm{MHzT}^{-1}$ to account for five different isotopes ${ }^{48}$ present in the dot $\left(^{113} \mathrm{In}\right.$, ${ }^{115} \mathrm{In},{ }^{69} \mathrm{Ga},{ }^{71} \mathrm{Ga},{ }^{75} \mathrm{As}$ ). We take $B_{z, \min }=0.06 \mathrm{~T}$ and use an average value $A_{\mathrm{hf}}=50 \mu \mathrm{eV}$ for all the isotopes ${ }^{49}$. The number of nuclei is taken to be $N=$ $4 \times 10^{4}$. The nuclear spin relaxation rate at a given magnetic field $B_{z}$ is then calculated by averaging over all parameter distributions to take into account the contributions of the individual nuclei in a QD.

Despite the simplifications, the model is in good agreement with experimental dependence $\Gamma_{\mathrm{N}}\left(B_{\mathrm{Z}}\right)$ in the $t_{\mathrm{B}}=37 \mathrm{~nm}$ sample (thin dashed line in Fig. $3 a$ ) and $t_{B}=42 \mathrm{~nm}$ sample (Supplementary Fig. 2). Qualitative 
comparison with Eq. (2) is possible in the high field limit where $\Gamma_{\mathrm{e}} \propto B_{z^{\prime}}^{4}$ $\left|M_{i j}\right|^{2} \propto B_{z}^{-2}$ and $v_{i j}^{2} \propto B_{z}^{2}$ leading to $\Gamma_{\mathrm{N}} \propto$ const, which agrees with the weak field dependence observed for all samples at $B_{z} \gtrsim 4 \mathrm{~T}$. By contrast, at fields $B_{z} \lesssim 4 \mathrm{~T}$, nuclear spin relaxation rate is determined by a combination of different factors prohibiting simple analytical description. Better description of the $\Gamma_{\mathrm{N}}\left(B_{z}\right)$ dependence, including the discrepancies with the thick-barrier $\left(t_{B}=52 \mathrm{~nm}\right)$ sample experiments, would require a more detailed model, which takes into account hyperfine fluctuations unrelated to electron spin flips, contributions of both noncollinear and direct hyperfine interaction, electron-nuclear spin feedback ${ }^{17}$, quadrupolar anticrossings $s^{36,37}$ of the individual nuclear spin levels and electronmediated nuclear-nuclear interactions ${ }^{15}$.

\section{DATA AVAILABILITY}

The data that support the findings of this study are available from the corresponding authors upon reasonable request.

Received: 31 October 2020; Accepted: 26 January 2021; Published online: 24 February 2021

\section{REFERENCES}

1. Kimble, H. J. The quantum internet. Nature 453, 1023 (2008).

2. Delteil, A. et al. Generation of heralded entanglement between distant hole spins. Nat. Phys. 12, 218 (2016)

3. Schwartz, I. et al. Deterministic generation of a cluster state of entangled photons. Science 354, 434-437 (2016).

4. Javadi, A. et al. Spin-photon interface and spin-controlled photon switching in a nanobeam waveguide. Nat. Nanotechnol. 13, 398-403 (2018).

5. Gangloff, D. A. et al. Quantum interface of an electron and a nuclear ensemble. Science 364, 62-66 (2019).

6. Chekhovich, E. A., da Silva, S. F. C. \& Rastelli, A. Nuclear spin quantum register in an optically active semiconductor quantum dot. Nat. Nanotechnol. 15, 999-1004 (2020).

7. Urbaszek, B. et al. Nuclear spin physics in quantum dots: An optical investigation. Rev. Mod. Phys. 85, 79-133 (2013).

8. Merkulov, I. A., Efros, A. L. \& Rosen, M. Electron spin relaxation by nuclei in semiconductor quantum dots. Phys. Rev. B 65, 205309 (2002).

9. Kroutvar, M. et al. Optically programmable electron spin memory using semiconductor quantum dots. Nature 432, 81-84 (2004).

10. Lu, C.-Y. et al. Direct measurement of spin dynamics in InAs/GaAs quantum dots using time-resolved resonance fluorescence. Phys. Rev. B 81, 035332 (2010).

11. Linpeng, $X$. et al. Longitudinal spin relaxation of donor-bound electrons in direct band-gap semiconductors. Phys. Rev. B 94, 125401 (2016).

12. Vaughan, M. P. \& Rorison, J. M. Model expressions for the spin-orbit interaction and phonon-mediated spin dynamics in quantum dots. Semicond. Sci. Technol. 33, 014001 (2017).

13. Huang, C.-W. \& Hu, X. Theoretical study of nuclear spin polarization and depolarization in self-assembled quantum dots. Phys. Rev. B 81, 205304 (2010).

14. Dreiser, J. et al. Optical investigations of quantum dot spin dynamics as a function of external electric and magnetic fields. Phys. Rev. B 77, 075317 (2008).

15. Latta, C., Srivastava, A. \& Imamoglu, A. Hyperfine interaction-dominated dynamics of nuclear spins in self-assembled InGaAs quantum dots. Phys. Rev. Lett. 107, 167401 (2011).

16. Houel, J. et al. Probing single-charge fluctuations at a GaAs/AlAs interface using laser spectroscopy on a nearby InGaAs quantum dot. Phys. Rev. Lett. 108, 107401 (2012).

17. Chekhovich, E. A. et al. Dynamics of optically induced nuclear spin polarization in individual InP/GalnP quantum dots. Phys. Rev. B 81, 245308 (2010).

18. Heiss, D. et al. Observation of extremely slow hole spin relaxation in selfassembled quantum dots. Phys. Rev. B 76, 241306 (2007).

19. Kim, J. H., Vagner, I. D. \& Xing, L. Phonon-assisted mechanism for quantum nuclear-spin relaxation. Phys. Rev. B 49, 16777-16780 (1994).

20. Dahan, P. \& Vagner, I. D. Nuclear spin relaxation rate of magnetic impurities in quantum Hall effect systems. Phys. Rev. B 72, 115328 (2005).

21. Kurzmann, A., Ludwig, A., Wieck, A. D., Lorke, A. \& Geller, M. Auger recombination in self-assembled quantum dots: quenching and broadening of the charged exciton transition. Nano Lett. 16, 3367-3372 (2016).

22. Atatüre, M. et al. Quantum-dot spin-state preparation with near-unity fidelity. Science 312, 551-553 (2006).
23. Warburton, R. J. et al. Optical emission from a charge-tunable quantum ring. Nature 405, 926-929 (2000)

24. Löbl, M. C. et al. Radiative Auger process in the single-photon limit. Nat. Nanotechnol. 15, 558-562 (2020).

25. Lyanda-Geller, Y. B., Aleiner, I. L. \& Altshuler, B. L. Charging effects on nuclear spin relaxation in quantum dots. J. Supercond. Nov. Magn. 16, 751-761 (2003).

26. Khaetskii, A. V. \& Nazarov, Y. V. Spin-flip transitions between Zeeman sublevels in semiconductor quantum dots. Phys. Rev. B 64, 125316 (2000).

27. Smith, J. M. et al. Voltage control of the spin dynamics of an exciton in a semiconductor quantum dot. Phys. Rev. Lett. 94, 197402 (2005).

28. Camenzind, L. C. et al. Hyperfine-phonon spin relaxation in a single-electron GaAs quantum dot. Nat. Commun. 9, 3454 (2018).

29. Kleemans, N. A. J. M. et al. Many-body exciton states in self-assembled quantum dots coupled to a Fermi sea. Nat. Phys. 6, 534-538 (2010).

30. Cunningham, J. E., Chiu, T. H., Tell, B. \& Jan, W. Atomic diffusion and surface segregation of $\mathrm{Si}$ in $\delta$-doped GaAs grown by gas source molecular beam epitaxy. J. Vac. Sci. Technol. B 8, 157-159 (1990).

31. De Franceschi, S. et al. Electron cotunneling in a semiconductor quantum dot. Phys. Rev. Lett. 86, 878-881 (2001).

32. Küng, B. et al. Quantum dot occupation and electron dwell time in the cotunneling regime. New J. Phys. 14, 083003 (2012).

33. Lu, J. et al. Nuclear spin-lattice relaxation in $n$-type insulating and metallic GaAs single crystals. Phys. Rev. B 74, 125208 (2006).

34. Erlingsson, S. I. \& Nazarov, Y. V. Hyperfine-mediated transitions between a Zeeman split doublet in GaAs quantum dots: The role of the internal field. Phys. Rev. B 66, 155327 (2002).

35. Woods, L. M., Reinecke, T. L. \& Lyanda-Geller, Y. Spin relaxation in quantum dots. Phys. Rev. B 66, 161318 (2002).

36. Chekhovich, E. A. et al. Structural analysis of strained quantum dots using nuclear magnetic resonance. Nat. Nanotechnol. 7, 646-650 (2012).

37. Bulutay, C. Quadrupolar spectra of nuclear spins in strained $\ln _{x} \mathrm{Ga}_{1-x}$ As quantum dots. Phys. Rev. B 85, 115313 (2012).

38. Maletinsky, P., Kroner, M. \& Imamoglu, A. Breakdown of the nuclear-spintemperature approach in quantum-dot demagnetization experiments. Nat. Phys. 5, 407-411 (2009).

39. Vladimirova, M. et al. Nuclear spin relaxation in $n$-GaAs: from insulating to metallic regime. Phys. Rev. B 95, 125312 (2017).

40. McNeil, J. A. \& Clark, W. G. Nuclear quadrupolar spin-lattice relaxation in some III-V compounds. Phys. Rev. B 13, 4705-4713 (1976).

41. Bechtold, A. et al. Three-stage decoherence dynamics of electron spin qubits in an optically active quantum dot. Nat. Phys. 11, 1005-1008 (2015).

42. Zhai, L. et al. Low-noise GaAs quantum dots for quantum photonics. Nat. Commun. 11, 4745 (2020).

43. Akimov, I. A., Feng, D. H. \& Henneberger, F. Electron spin dynamics in a self assembled semiconductor quantum dot: the limit of low magnetic fields. Phys. Rev. Lett. 97, 056602 (2006).

44. Ragunathan, G. et al. Direct measurement of hyperfine shifts and radio frequency manipulation of nuclear spins in individual CdTe/ZnTe quantum dots. Phys. Rev. Lett. 122, 096801 (2019).

45. Grovenor, C. Metallurgical reactions in Au/(In-Ge) ohmic contacts to GaAs. Thin Solid Films 104, 409-418 (1983).

46. Kuhlmann, A. V. et al. A dark-field microscope for background-free detection of resonance fluorescence from single semiconductor quantum dots operating in a set-and-forget mode. Rev. Sci. Instrum. 84, 073905 (2013).

47. Dalgarno, P. A. et al. Coulomb interactions in single charged self-assembled quantum dots: radiative lifetime and recombination energy. Phys. Rev. B 77 245311 (2008)

48. Harris, R. K., Becker, E. D., de Menezes, S. M. C., Goodfellow, R. \& Granger, P. NMR nomenclature. nuclear spin properties and conventions for chemical shifts (IUPAC Recommendations 2001). Pure Appl. Chem. 73, 1795-1818 (2001).

49. Checkhovich, E. A. et al. Element-sensitive measurement of the hole-nuclear spin interaction in quantum dots. Nat. Phys. 9, 74-78 (2013).

\section{ACKNOWLEDGEMENTS}

I.M.G. processed samples into Schottky diodes with input and advice from S. Kumar, B. Royal, I. Farrer, N. Babazadeh, K. Kennedy, A.U., and B. Harrison. E.C. acknowledges P. Patil, I. Farrer and J. Heffernan. Royal Society provided funding support to E.A.C. through University Research Fellowship and grant RGF\EA 180117 , and to G.G. and E. A.C. through grant RG150465. Experimental costs were part-funded through EPSRC grant EP/N031776/1. 


\section{AUTHOR CONTRIBUTIONS}

G.G., E.A.C. and A.U. developed time-resolved resonance fluorescence techniques. G. G., G.R., and E.A.C. developed nuclear spin lifetime measurement techniques. G.G. and E.A.C. conducted spin lifetime measurements. I.M.G., C.M. and E.A.C. conducted photoluminescence experiments. E.C. grew the samples. G.G. and E.A.C. analysed the data and wrote the manuscript. E.A.C. coordinated the project.

\section{COMPETING INTERESTS}

The authors declare no competing interests.

\section{ADDITIONAL INFORMATION}

Supplementary information The online version contains supplementary material available at https://doi.org/10.1038/s41534-021-00378-2.

Correspondence and requests for materials should be addressed to E.A.C.

Reprints and permission information is available at http://www.nature.com/ reprints
Publisher's note Springer Nature remains neutral with regard to jurisdictional claims in published maps and institutional affiliations.

Open Access This article is licensed under a Creative Commons Attribution 4.0 International License, which permits use, sharing, adaptation, distribution and reproduction in any medium or format, as long as you give appropriate credit to the original author(s) and the source, provide a link to the Creative Commons license, and indicate if changes were made. The images or other third party material in this article are included in the article's Creative Commons license, unless indicated otherwise in a credit line to the material. If material is not included in the article's Creative Commons license and your intended use is not permitted by statutory regulation or exceeds the permitted use, you will need to obtain permission directly from the copyright holder. To view a copy of this license, visit http://creativecommons. org/licenses/by/4.0/.

(c) The Author(s) 2021 